ARTICLE

Received 16 Apr 2015 | Accepted 29 Oct 2015 | Published 1 Dec 2015

DOI: $10.1038 /$ ncomms10062

OPEN

\title{
Production of butyrate from lysine and the Amadori product fructoselysine by a human gut commensal
}

Thi Phuong Nam Bui ${ }^{1}$, Jarmo Ritari ${ }^{2}$, Sjef Boeren ${ }^{3}$, Pieter de Waard ${ }^{4}$, Caroline M. Plugge ${ }^{1}$ \& Willem M. de Vos ${ }^{1,2,5}$

Human intestinal bacteria produce butyrate, which has signalling properties and can be used as energy source by enterocytes thus influencing colonic health. However, the pathways and the identity of bacteria involved in this process remain unclear. Here we describe the isolation from the human intestine of Intestinimonas strain AF211, a bacterium that can convert lysine stoichiometrically into butyrate and acetate when grown in a synthetic medium. Intestinimonas AF211 also converts the Amadori product fructoselysine, which is abundantly formed in heated foods via the Maillard reaction, into butyrate. The butyrogenic pathway includes a specific CoA transferase that is overproduced during growth on lysine. Bacteria related to Intestinimonas AF211 as well as the genetic coding capacity for fructoselysine conversion are abundantly present in colonic samples from some healthy human subjects. Our results indicate that protein can serve as a source of butyrate in the human colon, and its conversion by Intestinimonas AF211 and related butyrogens may protect the host from the undesired side effects of Amadori reaction products.

\footnotetext{
${ }^{1}$ Laboratory of Microbiology, Wageningen University, Dreijenplein 10, 6703 HB Wageningen, The Netherlands. ${ }^{2}$ Department of Basic Veterinary Medicine, University of Helsinki, Agnes Sjöberginkatu 2, FIN-00790 Helsinki, Finland. ${ }^{3}$ Laboratory of Biochemistry, Wageningen University, Dreijenlaan 3,6703 HA Wageningen, The Netherlands. ${ }^{4}$ Wageningen NMR Centre, Dreijenlaan 3, 6703 HA Wageningen, The Netherlands. ${ }^{5}$ Department of Bacteriology and Immunology, Haartmaninkatu 3, University of Helsinki, FIN-0014 Helsinki, Finland. Correspondence and requests for materials should be addressed to W.M.d.V. (email: willem.devos@wur.nl).
} 
O ur intestinal tract contains trillions of microbes that impact our health ${ }^{1}$. The metabolic capacity of these microbes is enormous ${ }^{2}$ and includes the production of short-chain fatty acids, such as butyrate that serves as a major source of energy for the intestinal enterocytes and signals to the host $^{3}$. Butyrogenic gut bacteria belong to Lachnospiraceae and Ruminococcaceae ${ }^{4}$, and recent studies showed an inverse correlation between these butyrogenic Firmicutes and inflammatory bowel disease, type 2 diabetes and various other disorders ${ }^{5,6}$. Moreover, there is considerable interest in these butyrogens, since butyrate reduces proinflammatory signals ${ }^{3}$ and has a protective role in maintaining a healthy $\operatorname{colon}^{7,8}$. Thus, it is of paramount importance to identify the microbes that are capable of producing butyrate and understand the mechanisms underlying its production.

Most butyrate produced in the human intestine is assumed to derive from carbohydrates, while some specialized bacteria have been found that convert lactate plus acetate into butyrate ${ }^{9,10}$. All of these produce butyrate via the acetyl-CoA pathway ${ }^{11}$ (converting acetyl-CoA to butyrate) that involves a complex cascade of reactions, in which butyrate CoA transferase (But) or butyrate kinase (Buk) are key enzymes. In a recent (meta)genome-based study, it was predicted that the But-mediated route was 10 -fold more abundant than that mediated by Buk, while these two enzymes showed high diversity among butyrogens ${ }^{12}$. Three other routes for butyrate synthesis, the glutamate, succinate and lysine pathways, have been described. On the basis of the distribution of the genes in the intestinal metagenome libraries, the acetylCoA pathway was the most prevalent followed by the lysine pathway. Yet, no intestinal bacterium has been reported that contained all genes coding for butyrogenesis from this amino acid ${ }^{13}$.

In addition to carbohydrates, proteins are crucial dietary compounds that are receiving increased attention to their use in special diets. Proteolytic enzymes in the upper intestinal tract generate amino acids that are rapidly taken up by the host, but also may reach the colon, partly depending on the intake. Moreover, protein recycling in the colon has been described, adding to the interest in colonic amino-acid fermentations. Amadori products (fructosamines), formed via non-enzymatic reactions between reducing sugars and free amino groups of proteins during the food heating process, are of significant interest as they have shown to be associated with the ageing process and several chronic diseases ${ }^{14}$. Fructoselysine is a key intermediate in the formation of advanced glycation endproducts (AGEs) ${ }^{14}$ and not yet any organism has been known to convert fructoselysine into butyrate. Only a few microbes have been reported to metabolize lysine into butyrate, and they derive from ecosystems other than the gut. A lysine degradation pathway was described over 50 years ago in Clostridium sticklandii isolated from black mud ${ }^{15}$. More recently, this pathway was detected in Clostridium subterminale SB4 isolated from sewage and Fusobacterium nucleatum isolated from the oral cavity, and eight genes encoding a lysine fermentation pathway were identified from the genome of $F$. nucleatum ${ }^{16}$. Several enzymes involved in butyrogenesis from lysine have been biochemically characterized ${ }^{17-20}$, but so far no intestinal microorganism has been found to contain the entire pathway in spite of the predicted presence of the lysine fermentation pathway in the human intestine ${ }^{13,16-18}$. Here we describe the butyrogenic conversion of lysine and the Amadori product fructoselysine by a novel Intestinimonas strain from a healthy volunteer and characterize its butyrate synthesis pathway by using a combined physiological, biochemical and proteogenomic approach.

\section{Results}

Lysine degradation by Intestinimonas AF211. In a search for new butyrogens, strain AF211 was isolated from the stool of a healthy subject using a mineral bicarbonate-buffered medium with lactate and acetate, under strict anoxic conditions (butyrate was found as the main end product). Strain AF211 contained two copies of the 16S rRNA gene that differed in a single nucleotide, and subsequent phylogenetic analysis showed that this strain belonged to clostridial cluster IV (Lachnospiraceae) of the Firmicutes phylum. Further analysis showed that this $16 \mathrm{~S}$ rRNA sequence was highly similar to that of the mouse intestinal isolate Intestinimonas butyriciproducens $\mathrm{DSM} 26588^{\mathrm{T}}$ (ref. 21), indicating that the newly isolated strain AF211 belongs to the genus Intestinimonas (Supplementary Fig. 1). However, strain Intestinimonas AF211 was a human isolate and showed physiological differences with I. butyriciproducens from mouse (to be reported elsewhere). Butyrate was found to be a major metabolite from all substrates where growth was observed (Supplementary Table 1). Intestinimonas AF211 hardly grew on glucose in mineral medium (generation time of 88 days), but its growth rate tripled in the presence of acetate and yeast extract. In media containing $20 \mathrm{mM}$ glucose with $20 \mathrm{mM}$ acetate and $2 \%$ yeast extract, Intestinimonas AF211 produced up to $4.4 \mathrm{mM}$ butyrate and trace amounts of lactate and ethanol (2-week incubation). Remarkably, the strain grew much better (generation time of $7.5 \mathrm{~h}$ ) in L-lysine but not in any other natural amino acids or D-lysine (Supplementary Table 1).

To study the capacity to convert lysine into butyrate, Intestinimonas AF211 was grown in bicarbonate-buffered medium containing L-lysine as the sole carbon and energy source. A total of $16.8 \pm 0.4 \mathrm{mM}$ lysine was converted into $14.2 \pm 0.6 \mathrm{mM}$ butyrate, $15.6 \pm 0.7 \mathrm{mM}$ acetate and $22.1 \pm 0.5 \mathrm{mM}$ $\mathrm{NH}_{3}$ when the cells reached the stationary phase after 2 days at $37^{\circ} \mathrm{C}$ (Fig. 1a). This suggests that part of the released ammonia is sequestered into proteins during anabolism of Intestinimonas $\mathrm{AF} 211$. Hence, we propose the fermentation reaction as: $\mathrm{C}_{6} \mathrm{H}_{14} \mathrm{O}_{2} \mathrm{~N}_{2}+2 \mathrm{H}_{2} \mathrm{O} \rightarrow \mathrm{C}_{4} \mathrm{H}_{8} \mathrm{O}_{2}+\mathrm{C}_{2} \mathrm{H}_{4} \mathrm{O}_{2}+2 \mathrm{NH}_{3}$. Clearly, Intestinimonas AF211 is capable of growing in defined media with L-lysine as sole carbon and energy source with a maximum growth rate of $0.1 \mathrm{~h}^{-1}$.

Growth of Intestinimonas AF211 on fructoselysine. As fructoselysine is a key intermediate in the formation of AGEs, we addressed its degradation by Intestinimonas AF211 and found it to be readily metabolized (Fig. 1b; generation time $24 \mathrm{~h}$ ). Butyrate, $\mathrm{NH}_{3}$ and lactate were formed as the main products. The conversion equation is proposed as: $\mathrm{C}_{12} \mathrm{H}_{24} \mathrm{O}_{7} \mathrm{~N}_{2}+2 \mathrm{H}_{2} \mathrm{O} \rightarrow 2$ $\mathrm{C}_{4} \mathrm{H}_{8} \mathrm{O}_{2}+2 \mathrm{NH}_{3}+1 \mathrm{CO}_{2}+1 \mathrm{C}_{3} \mathrm{H}_{6} \mathrm{O}_{3}$. We anticipate that the fructoselysine pathway includes the simultaneous degradation of both lysine and the sugar moiety in two branches (termed lysine pathway and acetyl-CoA pathway; Fig. 2). Some deviations in the butyrate/fructoselysine ratio were observed (Supplementary Tables 1 and 2). These may be attributed to the different environmental conditions but also be caused by the intracellular balance between the two branches of the fructoselysine pathway (Fig. 2). However, the initial concentration of acetate was predicted to be a major factor affecting the butyrate/fructoselysine ratio. We experimentally verified this and observed that in the presence of extra acetate, fructoselysine was completely converted into butyrate and $\mathrm{NH}_{3}$ (Supplementary Table 2) according to the equation: $\mathrm{C}_{12} \mathrm{H}_{24} \mathrm{O}_{7} \mathrm{~N}_{2}+\mathrm{C}_{2} \mathrm{H}_{4} \mathrm{O}_{2}+\mathrm{H}_{2} \mathrm{O} \rightarrow 3 \quad \mathrm{C}_{4} \mathrm{H}_{8} \mathrm{O}_{2}+2$ $\mathrm{NH}_{3}+2 \mathrm{CO}_{2}$. The growth rate in fructoselysine plus acetate was significantly higher than that in fructoselysine alone (Supplementary Table 2), which could be due to stimulation of butyrate production via external acetate, leading to an increased 
a

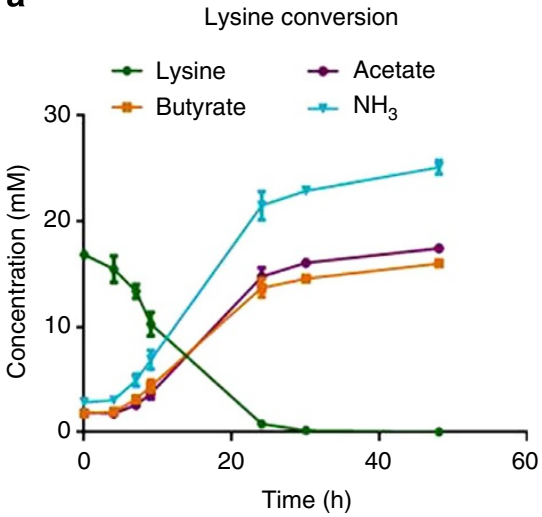

b

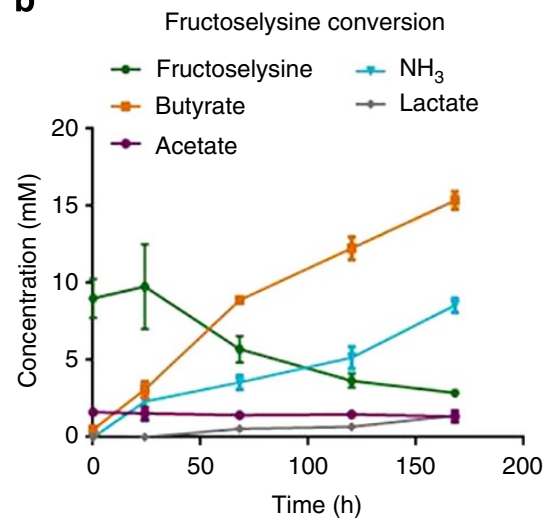

Figure 1 | L-lysine and fructoselysine conversion by strain Intestinimonas AF211 throughout time. Carbon recovery was $87 \%$ and $101 \%$ for the lysine (a) and fructoselysine (b) utilization, respectively. Exact concentrations of substrates and products and optical density values are included in Supplementary Table 1. Values are mean of biological duplicates. Error bars indicate s.d.

energy gain by generating a proton motive force via the membrane-associated Rnf complex and consequently, no extra $\mathrm{NADH}$ needed for lactate formation (Fig. 2). The influence of acetate on the growth of fructoselysine resembles the human gut environment where acetate is abundantly present ${ }^{11}$. Previously, the conversion of fructoselysine has been reported for a few bacteria, including Escherichia coli, which also converts psicoselysine $\mathrm{e}^{22,23}$. However, none of these bacteria are capable of butyrogenesis. To provide further support for the butyrogenic pathway we focused on lysine and glucose degradation by using a combined approach of nuclear magnetic resonance (NMR), proteogenome and enzyme activity assays.

Elucidation of the lysine degradation pathway using ${ }^{13} \mathrm{C}-\mathrm{NMR}$. To elucidate the butyrogenic pathway of Intestinimonas AF211, we applied in vivo ${ }^{1} \mathrm{H}$-decoupled ${ }^{13} \mathrm{C}$-NMR analysis of the culture supernatants of cells grown with L- $\left[2-{ }^{13} \mathrm{C}\right]$ lysine, L- $\left[6-{ }^{13} \mathrm{C}\right]$ lysine and lysine plus $\left[2-{ }^{13} \mathrm{C}\right]$ acetate as described previously ${ }^{24}$. Growth of Intestinimonas AF211 on $\mathrm{L}^{-}\left[6^{-13} \mathrm{C}\right]$ lysine resulted in its complete conversion into $\left[4-{ }^{13} \mathrm{C}\right]$ butyrate, $\left[2-{ }^{13} \mathrm{C}\right]$ acetate, $\left[2-{ }^{13} \mathrm{C}\right]$ butyrate and $\left[2,4-{ }^{13} \mathrm{C}\right]$ butyrate (Fig. 3a, left). Protondetected multiple-bond spectroscopy was also performed with the supernatant and based on the Heteronuclear Multiple-Bond Correlations (HMBCs) we could estimate the percentages of $\left[4-{ }^{13} \mathrm{C}\right]$ butyrate, $\left[2-{ }^{13} \mathrm{C}\right]$ butyrate and $\left[2,4-{ }^{13} \mathrm{C}\right]$ butyrate out of all labelled butyrate (Fig. 3b, left). Combination of this quantitative and kinetic analysis showed that at all time points the main product formed from $\mathrm{L}-\left[6-{ }^{13} \mathrm{C}\right]$ lysine was $\left[4-{ }^{13} \mathrm{C}\right]$ butyrate $(71 \%$; Fig. 3c), indicating that cleavage of lysine occurred between the $\mathrm{C} 2$ and $\mathrm{C} 3$ residues. Both $\left[4-{ }^{13} \mathrm{C}\right]$ butyrate, $\left[2-{ }^{13} \mathrm{C}\right]$ butyrate and $\left[2,4-{ }^{13} \mathrm{C}\right]$ butyrate were detected in the cells grown in lysine plus $\left[2-{ }^{13} \mathrm{C}\right]$ acetate (Fig. 3a,b, right). This is indicative of simultaneous operation of the acetyl-CoA and lysine degradation pathways (Fig. 2) and explained the formation of minor amounts of $\left[2-{ }^{13} \mathrm{C}\right]$ acetate, $\left[2-{ }^{13} \mathrm{C}\right]$ butyrate and $\left[2,4-{ }^{13} \mathrm{C}\right]$ butyrate in $\mathrm{L}-[6-$ $\left.{ }^{13} \mathrm{C}\right]$ lysine. Altogether, these data provide molecular evidence that the lysine pathway was substantially active and generated the intermediates for the acetyl-CoA pathway in Intestinimonas AF211. Similar results supporting the simultaneous operation of the two pathways were obtained by using D,L- $\left[2-{ }^{13} \mathrm{C}\right]$ lysine in growing cells of Intestinimonas AF211, and the NMR analysis also confirmed the exclusive selectivity for L-lysine (Supplementary Fig. 2). High-performance liquid chromatography (HPLC) analysis confirmed the nearly complete conversion of lysine to equimolar amounts of butyrate and acetate as indicated above (Fig. 1a). The molecular events that explain the observed isotopomers derived from $\left[{ }^{13} \mathrm{C}\right]$ lysine were reconstructed (Supplementary Fig. 3) and the deduced metabolic pathway revealed 10 enzymatic reactions that were further characterized by genomic, proteomic and enzyme studies (Fig. 2).

Proteogenomic analysis of the metabolic pathways. The complete genome of Intestinimonas AF211 was determined using single-molecule next-generation sequencing revealing a circular chromosome of $3,376,476 \mathrm{bp}$, predicted to encode 3,359 genes (NCBI accession number CP011307; to be reported elsewhere). Gene candidates for the fructoselysine and lysine metabolic pathways and linked energy-generating conversions were identified in the annotated genome of Intestinimonas AF211 (Fig. 2 and Supplementary Fig. 4). These included an operon-like cluster (AF_00949-00955) with genes for fructoselysine and psicoselysine uptake and degradation ${ }^{14}$ and a lysine utilization operon (AF_00976-00982) coding for six key enzymes involved in converting lysine into butyrate (Supplementary Fig. 4).

To confirm the presence and gene expression of the lysine degradation pathway in Intestinimonas AF211, proteins extracted from cells grown on either lysine or glucose and acetate were analysed by semi-quantitative proteomics (Supplementary Fig. 4 and Supplementary Data 1). Intestinimonas AF211 was found to produce all proteins involved in the conversion of glucose and acetate into butyrate and employed the acetyl-CoA pathway, similar to other members of Ruminococcaceae and Lachnospiraceae $^{8}$. Moreover, the proteins involved in the lysine pathway (encoded by the cluster AF_00976-00982) were not only highly abundant but also differentially and $\sim 10$-fold induced during growth on lysine compared with glucose and acetate, except for 3-aminobutyryl-CoA ammonia-lyase (encoded by AF_00976), which was not detected. The latter protein is rather small $(14 \mathrm{kDa})$ and may have been lost during the protein extraction. Another annotated Kce candidate (AF_02981) was found to be reduced 10 -fold during the growth in lysine and is present in very low level, pointing to another function rather than being involved in the lysine pathway. Two predicted 3-ketoacyl-CoA thiolases (AF_03338 and AF_00601) were 1.3-fold and 1.8-fold more abundant in lysine-grown cells than glucose and acetate-grown cells. AF_03338 is located in the same gene cluster operon with AtoD-A (AF_03339-03340; see below), which is a lysineinducible key enzyme in the lysine pathway ${ }^{19}$. Thus, AF_03338 is likely to be co-expressed under lysine growth conditions, while 


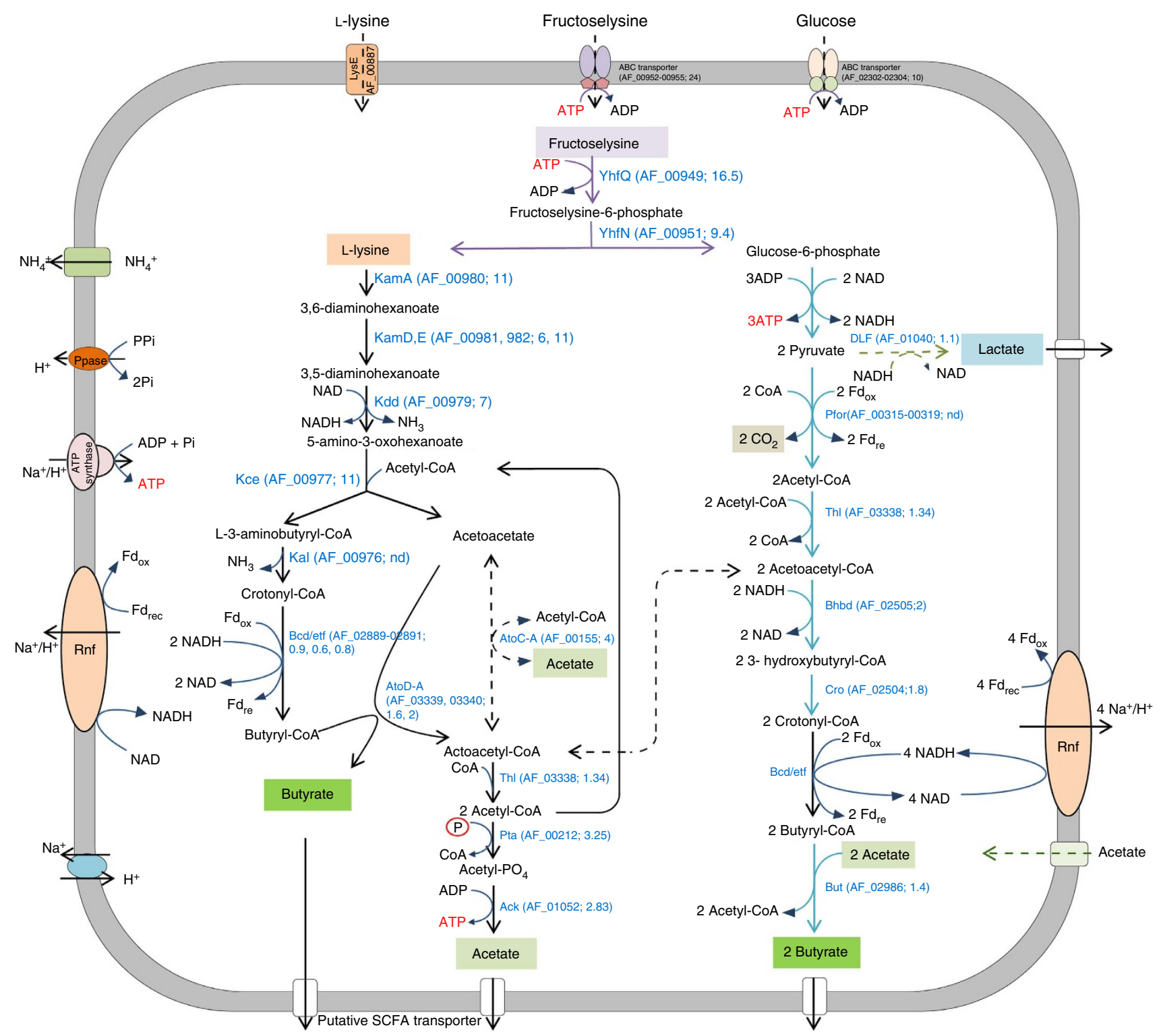

Figure 2 | Model for fructoselysine metabolism in Intestinimonas AF211. The locus tag and fold induction of the proteins based on the proteomic data are indicated in the brackets. Fd, ferredoxin; ND, not detected; Ppase, pyrophosphatase; Rnf, proton pumping Rnf cluster. According to our model, fructoselysine is taken up by $A B C$ transporter (AF_00952-00955), which is phosphorylated by fructoselysine kinase (AF_00949) to form fructoselysine-6-phosphate that is subsequently cleaved by fructoseamine deglycase (AF_00951) into lysine and glucose-6-phosphate (indicated by the purple arrows). Lysine is then degraded via the lysine pathway (black arrows) while glucose-6-phosphate is metabolized via glycolysis and the acetyl-CoA pathway (blue arrows) as described in the text (Supplementary Fig. 4). There are various links between the lysine and acetyl-CoA pathways and the one at the level of acetoacetyl$\mathrm{CoA}$, involving acetate to generate acetoacetate and acetyl-CoA, is indicated (dashed black arrows). For simplicity, only the key reactions are shown and other links or the conversion of acetyl-CoA formed via acetyl-CoA pathway to acetate have been omitted. Fructoselysine, lysine, butyrate, acetate, lactate and $\mathrm{CO}_{2}$ are indicated in different colours highlighting their distinctive positions in the pathways. ATP is in red to indicate reactions that either require or generate energy via substrate-level phosphorylation and electron transport chain. Lactate formation is dependent on the amount of exogenous acetate and redox state (hence both lactate formation and acetate uptake are indicated by dashed green arrows). Therefore, the overall fructoselysine stoichiometry depends on the presence or absence of exogenous acetate as well as environmental conditions and activity of biosynthetic pathways. In the presence of acetate (such as in the human colon), fructoselysine is converted in approximately three butyrate (two butyrate are formed via acetyl-CoA pathway and one butyrate through the lysine pathway), while no lactate is produced; however, when no exogenous acetate is present, fructoselysine is converted into approximately two butyrate and one lactate (see Supplementary Table 2 for details).

the product of AF601 might participate in the simultaneous conversion of acetate into butyrate. Several predicted butyrylCoA dehydrogenase $(b c d)$ genes were detected in the genome but only one of these (AF_02889) formed an operon-like cluster with the Etf complex genes ${ }^{25}$ (AF_02890-02891). The reactions catalysed by ferredoxin oxidoreductase plus the exergonic
NADH-dependent reduction of crotonyl-CoA to butyryl-CoA show a possible chemi-osmotic energy conservation via the Rnf comple ${ }^{26}$. This is essential under all growth conditions and hence the proteins encoded by this gene cluster were always detected (Fig. 2). The phosphate transacetylase (AF_00212) and acetate kinase (AF_01052) were found to be induced $\sim 3$-fold in 
a

$\left[6^{-13} \mathrm{C}\right] \mathrm{ysine}$

$72 \mathrm{~h}$

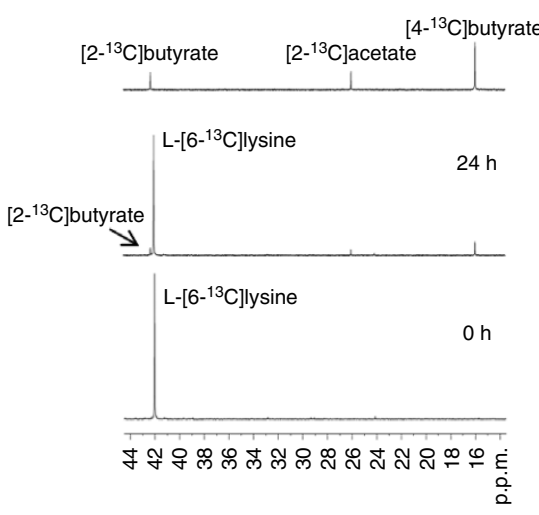

Lysine plus $\left[2-{ }^{13} \mathrm{C}\right]$ acetate

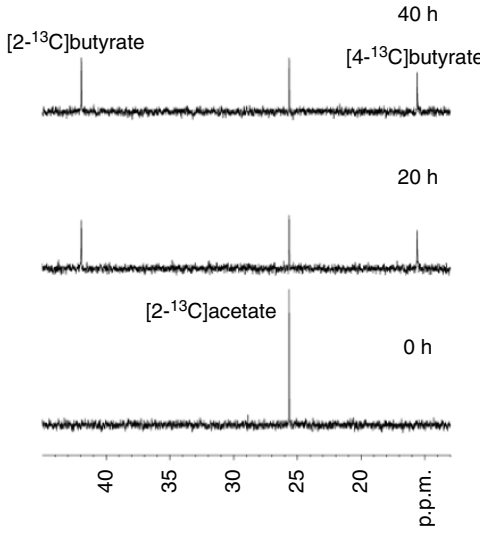

b
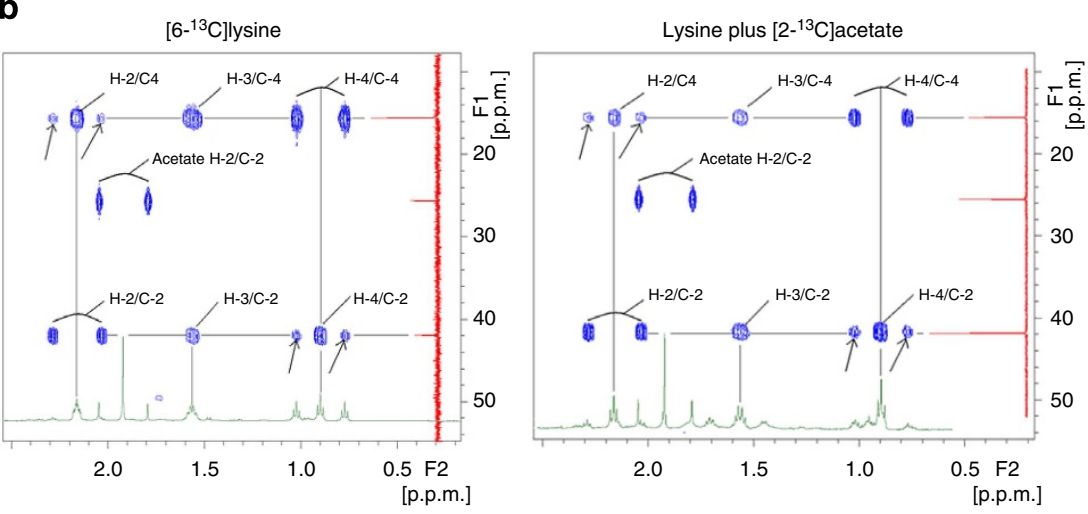

C

\begin{tabular}{lccc}
\hline \multirow{3}{*}{ Substrates } & \multicolumn{3}{c}{ Products (\%) } \\
\cline { 2 - 4 } & {$\left[4-{ }^{-13} \mathrm{C}\right]$ butyrate } & {$\left[2-{ }^{-13} \mathrm{C}\right]$ butyrate } & {$\left[2,4-{ }^{13} \mathrm{C}\right]$ butyrate } \\
\hline$\left[6-{ }^{13} \mathrm{C}\right]$ lysine & 71 & 22.5 & 6.4 \\
Lysine $+\left[2-{ }^{13} \mathrm{C}\right]$ acetate & 39 & 55 & 6 \\
\hline
\end{tabular}

Figure 3 | Elucidation of lysine pathway via ${ }^{\mathbf{1}} \mathbf{H}$-decoupled ${ }^{13} \mathbf{C}-\mathbf{N M R}$ spectrum and $\mathbf{2 D}$ HMBC spectrum. (a) High-resolution ${ }^{1} \mathrm{H}$-decoupled ${ }^{13} \mathrm{C}-\mathrm{NMR}$ spectra showing $L-\left[6-{ }^{13} \mathrm{C}\right]$ lysine ${ }^{13} \mathrm{C}$-labelled fermentation products. $\left[2-{ }^{13} \mathrm{C}\right]$ butyrate, $\left[2-{ }^{13} \mathrm{C}\right]$ acetate and $\left[4-{ }^{13} \mathrm{C}\right]$ butyrate had a chemical shift of 42.33 , 25.99 and 15.95 p.p.m., respectively. (b) $2 \mathrm{D} \mathrm{HMBC}$ spectrum for $\left[6-{ }^{13} \mathrm{C}\right]$ lysine is shown. (c) Percentages of labelled butyrate fractions (see Supplementary Figs 2 and 3 for more details).

lysine-grown cells compared with glucose and acetate-grown cells, indicative of the involvement of these two enzymes in the lysine pathway. Remarkably, proteome analysis revealed the fructoselysine operon-like genes to be induced up to 25 -fold during growth on lysine (Supplementary Fig. 4 and Supplementary Data 1), most likely due to a high amount of lysine, which was also an intermediate in the fructoselysine pathway (Fig. 2).

Phylogeny of CoA transferases and enzyme activity. A crucial reaction involved in butyrate formation from lysine is the transfer of a CoA moiety from one molecule to another, catalysed by members of the CoA transferase family. Remarkably, analysis of the genome of Intestinimonas AF211 predicted the presence of 14 such enzymes, and a phylogenetic tree was generated based on these and CoA transferases from intestinal bacteria and environmental isolates (Fig. 4a). These include the CoA transferases AtoD-A, 4Hbt and But, for which experimental evidence for their involvement in butyrate synthesis has been described in several anaerobes, including Clostridium SB4, Roseburia spp., Faecalibacterium prausnitzii and Clostridum acetobutylicum $^{8,19,27}$. Five well-separated clades of enzymes could be distinguished. One clade included the But enzymes from the main butyrate producers in the human intestine, belonging to the Lachnospiraceae, and Ruminococcaceae ${ }^{4}$ that are all capable of butyrogenesis from glucose or lactate plus acetate ${ }^{8,28}$. The Intestinimonas AF211 enzyme encoded by AF_02986 belonged to this But clade and was detected in the proteome under both growth conditions, indicating that it participated in the acetyl-CoA pathway rather than 4-aminobutyrate or glutamate pathways. The second clade harboured the $4 \mathrm{Hbt}$ enzymes and included several predicted CoA transferases of Intestinimonas AF211 but none was detected at the protein level. The third and fourth clades held the $\alpha$ and $\beta$ subunits of the AtoD-A enzymes, respectively, that are encoded by two juxtaposed genes in 
a

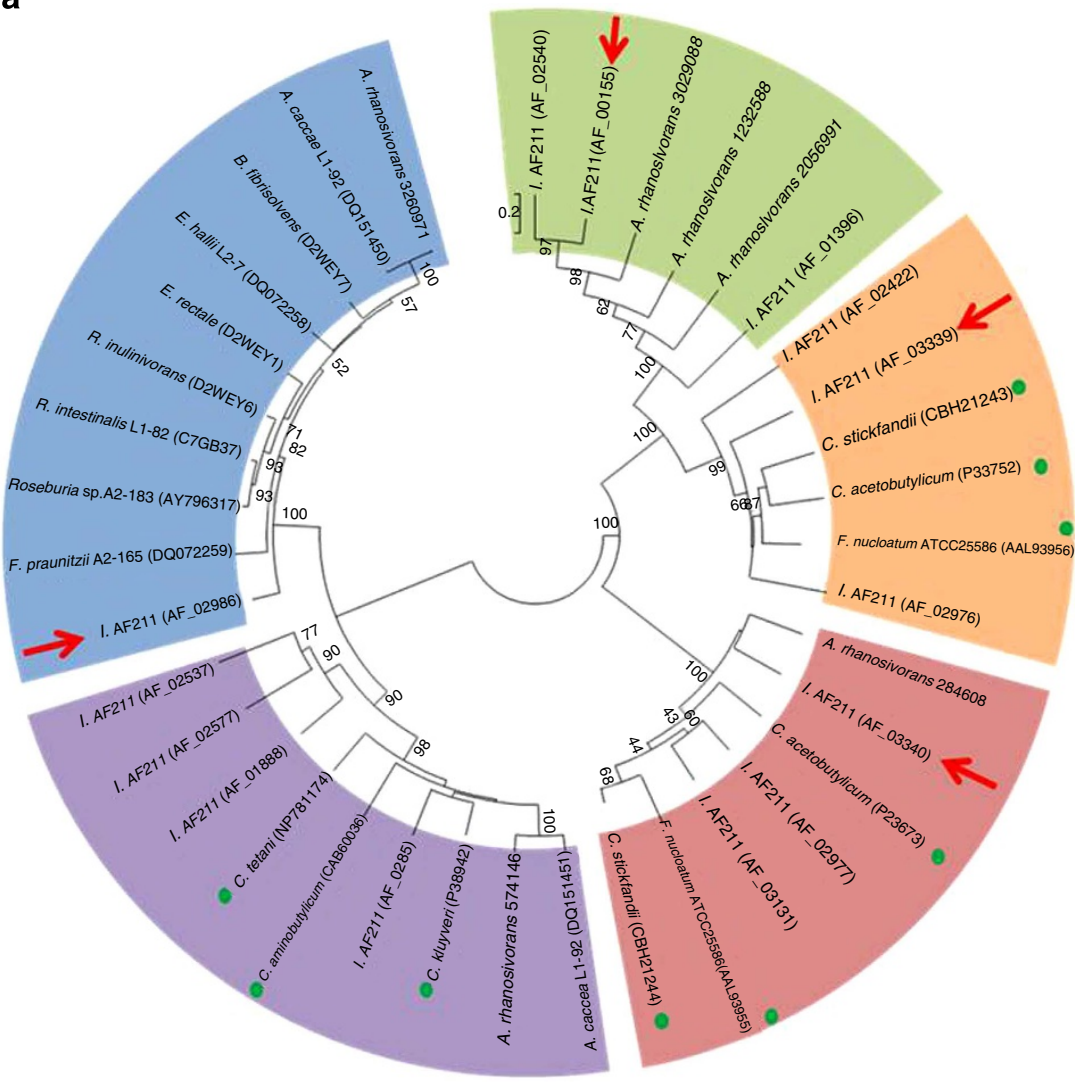

b
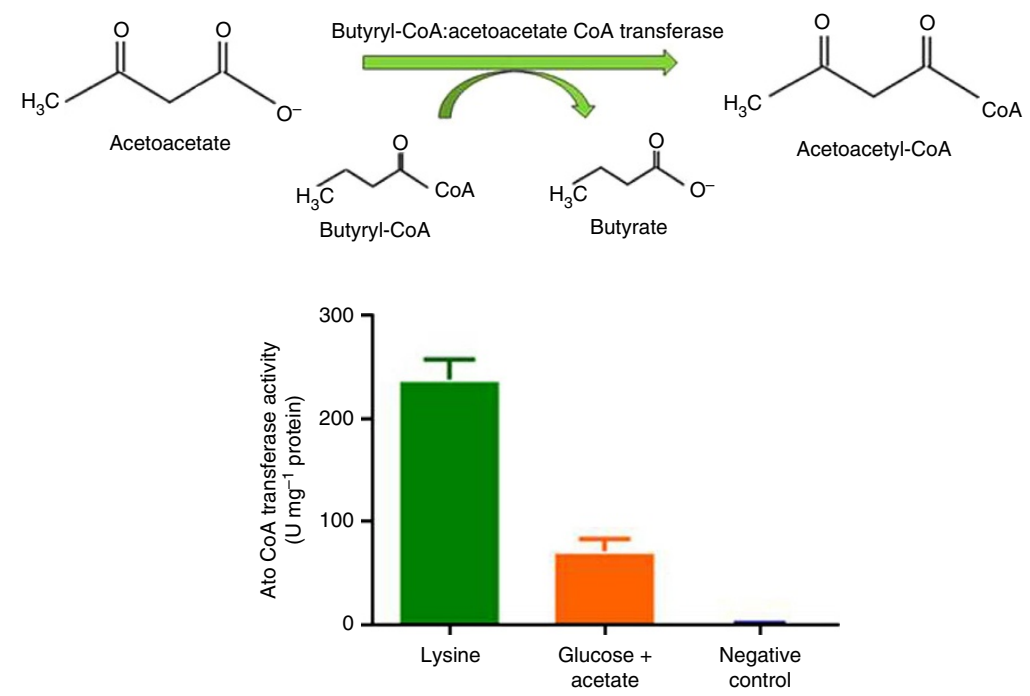

Figure 4 | Phylogeny of CoA transferase and enzyme activity. (a) Phylogenetic tree of predicted CoA transferases from Intestinimonas AF211 (bold) and other representative anaerobes. The tree was based on sequences from butyryl-CoA:acetate CoA transferase (But, in blue), butyryl-CoA:4-hydroxybutyrate CoA transferase (4 Hbt, in purple), butyryl-CoA:acetoacetate CoA transferase (Ato) alpha subunit (AtoD, in orange), beta subunit (AtoA, in brown) and acetyl-CoA:acetoacetate CoA transferase (AtoC, in green), respectively. Green dots indicate non-intestinal isolates. Intestinimonas AF211 proteins induced during growth on lysine are indicated by the red arrows. (b) Butyryl-CoA:acetoacetate CoA transferase activity in crude cell extracts. Each measurement was performed with biological duplicates and 4-6 replicate measurements. Values represent mean of replicates. Error bars indicate s.d.'s. Green and red bars represent the enzyme activity of Intestinimonas AF211 grown in lysine and glucose plus acetate, respectively; blue bar is the negative control with A. rhamnosivorans DSM26241 grown on glucose, which is not capable of lysine fermentation.

the well-studied anaerobes: F. nucleatum, C. sticklandii and C. acetobutylicum ${ }^{29-33}$. Within these two AtoD-A subunit clades, three gene pairs of Intestinimonas AF211 were clustered. However, only one of these, encoded by AF_03339-03340, was highly abundant and induced two-fold under lysine growth conditions, indicative of its involvement in butyrate formation via the lysine pathway (Fig. 2). A fifth cluster of acetylCoA:acetoacetate CoA transferases (AtoC) contained enzymes not only from Intestinimonas AF211 (indicated by AF_00155, AF_02540 and AF_01396) but also from Anaerostipes 
rhamnosivorans, which is capable of butyrogenesis from glucose as well as from acetate plus lactate ${ }^{34}$. The four-fold protein induction of AtoC (AF_00155) during growth on lysine indicated the involvement of this CoA transferase in lysine degradation. We propose its role to be the balancing of acetyl-CoA, released from the acetyl-CoA pathway and providing either additional acetoacetate or acetoacetyl-CoA, in line with the simultaneous activity of the lysine and acetyl-CoA pathway during butyrogenesis from lysine (see above).

To further provide support for the pivotal conversion catalysed by AtoD-A, its activity in Intestinimonas AF211 was studied by incubating cell-free extracts under anaerobic conditions with acetoacetate and butyryl-CoA, and monitoring the production of acetoacetyl-CoA ${ }^{19,30}$. We found reproducible and high activity of this enzyme activity in cells grown on lysine (237 units per mg protein), which was 3.5-fold reduced in cells grown with glucose and acetate ( 71 units per mg protein; Fig. 4b). As the observed activity induction coincided with the protein quantitation from the proteome analysis, we deduce that AtoD-A (encoded by AF_03339-03340) is probably the butyryl-CoA:acetoacetate CoA transferase involved in the butyrogenic lysine pathway.

Intestinimonas and fructoselysine genes in the human gut. As metagenome analysis indicated that the lysine pathway has high abundance in the human intestine ${ }^{13}$, we studied the presence of Intestinimonas related to AF211 in a series of healthy subjects by using a specific $16 \mathrm{~S}$ rRNA-based quantitative PCR (qPCR) (Supplementary Table 3). In 5 out of 10 subjects, $0.2-10 \%$ of the 16S rRNA sequences were derived from Intestinimonas spp. since all qPCR products showed the expected nucleotide sequence. From faecal DNA of the remaining five subjects, amplicons were generated, but sequence analysis showed four of them to derive from Ruminococcus spp. as their 16S rRNAs fortuitously amplified with the used primers, indicating it is not possible to correctly estimate the level of Intestinimonas spp. in these subjects (Supplementary Table 3 ). When analysing the deep metagenome information obtained in 65 subjects characterized in the Human Microbiome Project ${ }^{35}$, we could identify many of the genes for lysine degradation in over half of the subjects but the genes involved in fructoselysine degradation were only observed in half a dozen individuals as indicated by the presence of the key gene fructoselysine kinase (Supplementary Fig. 5).

The observed abundance and prevalence of Intestinimonas spp. level is in good agreement with the metagenome-predicted presence of the lysine degradation pathway ${ }^{13}$, suggesting that Intestinimonas is the key species converting lysine and fructoselysine into butyrate in the human gut. This is supported by the recent isolation of a similar but antibiotic-resistant strain from a healthy subject ${ }^{36}$. However, it is evident that not all humans have intestinal metagenomes that are equipped with genes for the conversion of fructoselysine, the major Amadori product.

\section{Discussion}

Here we describe the isolation of a butyrate-producing bacterium, Intestinimonas AF211, abundantly present in the intestine of some humans. The bacterium can use fructoselysine, a key intermediate in the formation of AGEs, as sole carbon and energy source by converting this into mainly butyrate and $\mathrm{NH}_{3}$. The predicted fructoselysine pathway includes the simultaneous degradation of both lysine and sugar moiety. By determining the metabolic route of the ${ }^{13} \mathrm{C}$-labelled lysine conversion by $\mathrm{NMR}$, in combination with enzyme measurements and proteogenomic analysis, the butyrogenic pathway from lysine was fully elucidated and also indicated the presence of the fructoselysine pathway in Intestinimonas AF211. The use of fructoselysine as carbon and energy source for butyrogenesis is unique, and Intestinimonas AF211 is the first intestinal bacterium to harbour the complete pathway for the conversion of lysine into butyrate (Fig. 1), previously predicted based on metagenomic data ${ }^{13}$. Lysine is an essential amino acid that is cleaved from dietary proteins by pancreatic trypsin, producing peptide chains with a C-terminal arginine or lysine residue ${ }^{37}$, The generated lysinecontaining peptides can be utilized via the proteolytic activity of Intestinimonas AF211 as evidenced by its growth on different protein-derived substrates (Supplementary Table 4) and the several-fold induction of various aminopeptidases under lysine growth condition (Supplementary Table 5). Collectively, one can consider the butyrogenic conversion of lysine by Intestinimonas AF211 as a specific example of host-microbe interactions.

Degradation of L-lysine was previously observed in complex media by F. nucleatum, suggesting that this Gram-negative bacterium and potential pathogen can use L-lysine as energy source but its use as carbon source is not clear ${ }^{16,32,38}$. C. sticklandii uses L-lysine as an electron donor in the Stickland reaction and lysine was only degraded in the stationary phase when other amino acids were depleted ${ }^{29}$. Despite the fact that a few bacteria have been reported to degrade lysine to butyrate, recent (meta) genomic analysis, which included genomes from these isolates, indicated that none of the genomes analysed had genes for the entire pathway ${ }^{13}$. In addition, E. coli and Bacillus subtilis were found to be capable of degrading fructoselysine but none of them produces butyrate ${ }^{14}$. However, Intestinimonas AF211 contained all genes involved in degradation of fructoselysine to butyrate (Fig. 2) while proteomic analysis revealed that these genes were induced under the lysine growth conditions.

Remarkably and unlike other butyrogenic bacteria, Intestinimonas AF211 was found to contain over a dozen genes coding for CoA transferases (Fig. 4a). We hypothesize that this may help the bacterium to be more flexible to act on a broad range of substrates. The integrated analysis of the Intestinimonas AF211 genome, proteome and activity measurements revealed that a specific acetoacetyl-CoA transferase AtoD-A (AF_03339-03340) was abundantly expressed under lysine degradation conditions and hence predicted to be the key enzyme involved in butyrate synthesis. As this is the first described lysine pathway in the intestinal ecosystem, the lysine utilization operon and the identified AtoD-A genes may have application as markers for butyrogenesis from lysine.

An important corollary of the abundant presence of lysine-degrading butyrogenic Intestinimonas AF211 and relatives in the intestine of some humans is the fact that amino acids can serve as source of butyrate formation. Since butyrate has presumed health benefits as an energy source for colonocytes and is a vital molecule to maintain intestinal integrity, this may nuance suggestions that protein fermentation, notably in the distal colon, has a negative health impact ${ }^{39}$. Remarkably, we observed that Intestinimonas AF211 is also capable of growth on fructoselysine as sole carbon and energy source resulting in the conversion of fructoselysine into butyrate (Supplementary Table 1). The Amadori product fructoselysine is abundant in cooked foods and is formed via the non-enzymatic Maillard reaction of reducing sugars and amino acids during the heating process. As humans are unique in the consumption of cooked products at a large scale, it would be of interest to determine whether the fructoselysine gene cluster detected in Intestinimonas AF211 has been recently acquired and is not present in the gut microbes of other primates. The production of fructoselysine from cooked foods has various impacts, including the loss of essential amino acids and a reduced protein digestibility. 
Fructoselysine is key product leading to the formation of AGEs in the human body that have been associated with chronic diseases and development of diabetes complication ${ }^{40-42}$. Moreover, recent food interventions in mice showed the deleterious effect of AGEs on these and other diseases ${ }^{43}$. The butyrogenic conversion of fructoselysine by Intestinimonas AF211 illustrates the important role of this anaerobe in the human intestinal tract, whereas the observation that some but not all human carry genes for fructoselysine degradation indicates the potential for specific interventions with the newly discovered strain. In conclusion, our study underlines the need for cultivating novel microbes to get a comprehensive understanding of the intestinal metabolic processes and the beneficial effect on the human host. In addition, Intestinimonas AF211 and related bacteria may play an important role in the intestinal tract by maintaining protein balance and gut homeostasis.

\section{Methods}

Enrichment, isolation and growth. Strain Intestinimonas AF211 was isolated from the stool of a healthy adult. The faecal sample was enriched in an anaerobic bicarbonate-buffered mineral salt medium ${ }^{44}$ containing $40 \mathrm{mM}$ lactate and $40 \mathrm{mM}$ acetate as energy and carbon source. The head space was filled with $\mathrm{CO}_{2} / \mathrm{N}_{2}(1: 4)$ at $1.5 \mathrm{~atm}$ and incubation was at $37^{\circ} \mathrm{C}$. Subsequently, the enrichment culture was transferred to reinforced clostridium medium (RCM, Difco) in serial dilutions and plated at least three times on RCM agar, which resulted in an axenic culture. The purity of the strain, designated as strain AF211, was confirmed by 16S rRNA gene sequencing and microscopy. The strain was routinely maintained in RCM medium at $37^{\circ} \mathrm{C}$. A phylogenetic tree of the $16 \mathrm{~S}$ rRNA of Intestinimonas AF211 (GenBank accession JX273469) and closely related strains was constructed as described previously $^{34}$ (Supplementary Fig. 1).

For physiological characterization, different amino acids and amino-acid derivatives were tested including $20 \mathrm{mM}$ of L-lysine, fructoselysine (USBiological, USA), glutamate, glutamine, glycine, proline, arginine, aspartate and methionine, which were added into the bicarbonate-buffered medium from $1 \mathrm{M}$ sterile anoxic stock solutions. For sugar utilization $20 \mathrm{mM}$ glucose plus $20 \mathrm{mM}$ acetate were added into the bicarbonate-buffered medium from $1 \mathrm{M}$ stock solutions. The growth was determined via product formation by HPLC ${ }^{34}$ and optical density measurement by a spectrophotometer at a wavelength of $600 \mathrm{~nm}$. Generation times were calculated employing Gompertz modelling ${ }^{45}$.

Analytical methods. Lysine was quantified on a HPLC using a Polaris C18-A column (Agilent) running at $45^{\circ} \mathrm{C}$ and an ultraviolet-visible detector at wavelength of $436 \mathrm{~nm}$. Flow rate was $0.5 \mathrm{ml} \mathrm{min}^{-1}$. An eluent mobile phase consisted of $24 \mathrm{mM}$ acetic acid:8\% acetonitrile ( $\mathrm{pH}$ 6.6) as solvent A and acetonitrile:2-propanol $(60: 40)$ as solvent B. The gradient elution was used from $95 \%$ eluent A and $5 \%$ eluent B to $25 \%$ eluent A and $75 \%$ eluent B for first $15 \mathrm{~min}$. The column was subsequently washed with $100 \%$ eluent B for 7 min before the next sample injection. Norleucine $(4 \mathrm{mM})$ was used as internal standard. The volatile fatty acid formation was measured on a Thermo Scientific Spectra HPLC system equipped with an Agilent Metacarb $67 \mathrm{H} 300 \times 6.5-\mathrm{mm}$ column kept at $37^{\circ} \mathrm{C}$ and running with $10 \mathrm{mM}$ arabinose as an eluent. The detector was a refractive index detector. The eluent flow was $0.8 \mathrm{ml} \mathrm{min}-1$. Gas production was performed as previously described ${ }^{46}$. All analyses were performed in duplicate. Ammonium was determined using Spectroquant test kit according to the manufacture's instruction. Fructoselysine was separated by ion-exchange chromatography and quantified by post column reaction with ninhydrin using photometric detection at $570 \mathrm{~nm}$ (International Organization for Standardization (ISO) 13903).

Nuclear magnetic resonance. Strain AF211 was cultivated in a bicarbonatebuffered medium containing $20 \mathrm{mM}$ of $\left[2-{ }^{13} \mathrm{C}\right]_{\mathrm{L}}$-lysine or $\left[6-{ }^{13} \mathrm{C}\right]_{\mathrm{L}}-\mathrm{lysine}$ or lysine plus $\left[2-{ }^{13} \mathrm{C}\right]$ acetate. The growth conditions were as described above. ${ }^{13} \mathrm{C}$-labelled lysine was purchased from Campro Scientific (Veenendaal, The Netherlands). Samples were taken from an overnight culture and centrifuged at $10,000 \mathrm{~g} \cdot \mathrm{D}_{2} \mathrm{O}$ $(50 \mu \mathrm{l} ; 99.9$ atom\%, Sigma Aldrich) was added to the supernatants $(0.5 \mathrm{ml})$ and subsequently transferred in NMR tubes (Campro Scientific). ${ }^{1} \mathrm{H}$-decoupled ${ }^{13} \mathrm{C}$ NMR spectra were recorded at a probe temperature of $300 \mathrm{~K}$ on a Bruker AvanceIII-500 spectrometer located at the Wageningen NMR Centre (WNMRC), Wageningen, the Netherlands. Chemical shifts are expressed in p.p.m. relative to the C- 6 of added $\left[6-{ }^{13} \mathrm{C}\right]$ lysine at 41.75 p.p.m., added $\left[2-{ }^{13} \mathrm{C}\right]$ lysine at 57.19 p.p.m., formed $\left[2{ }^{13} \mathrm{C}\right]$ butyrate at 42.33 p.p.m., formed $\left[4-{ }^{13} \mathrm{C}\right]$ butyrate at 15.95 p.p.m. and formed $\left[2-{ }^{13} \mathrm{C}\right]$ acetate at 25.99 p.p.m. (Biological Magnetic Resonance Data Bank, http://www.bmrb.wisc.edu/metabolomics/metabolomics_standards). For the HBMC spectra 400 experiments of 8 scans were recorded resulting in a measuring time of $50 \mathrm{~min}$, using a standard Bruker pulse sequence. The products were identified based on chemical shifts as compared with database as mentioned above.
In the HMBC experiment no decoupling is used (Fig. 3b). Therefore, the single-bond couplings will result in double cross-peak split by the large single-bond coupling. The active coupling between 5 and $10 \mathrm{~Hz}$ of two- and three-bond couplings remains within the width of a cross-peak in a HMBC spectrum, resulting in single cross-peaks. When the carbon attached to the proton involved in a three-bond coupling is also enriched, this cross-peak will be split by the large single-bond coupling with this carbon. These splittings are visible as extra peaks next to the single cross-peaks between $\mathrm{H}-2$ and C- 4 and between $\mathrm{H}-4$ and C-2 (arrows in the figures), indicating double enrichment of both C-2 and C-4. The calculation was shown in Fig. 3c. Both the split and non-split HMBC cross-peaks with $\mathrm{C}-4$ at $\mathrm{H}-2$ and with $\mathrm{C}-2$ at $\mathrm{H}-4$ were integrated. The integrals of the split and non-split cross-peaks at a single proton are comparable, since they are based on the same three-bond couplings and experience similar relaxation behaviour. The split cross-peaks of H-2 with C-4 and of H-4 with C-2 show a small difference due to sensitivity of the HMBC experiment for difference in relaxation and difference in coupling values. Since these split cross-peaks refer to the same population of $\left[2,4-{ }^{13} \mathrm{C}\right]$ butyrate, the factor needed to equalize them can also be used to correct the value of the non-split values, thus revealing the percentages of all three possible fractions of labelled butyrate.

Proteomics. The protein abundances in cultures growing with different substrates were investigated with liquid chromatography-mass spectrometry/mass spectrometry. Strain AF211 was grown in triplicate in $500 \mathrm{ml}$ bicarbonate-buffered medium containing with $20 \mathrm{mM}$ lysine and $40 \mathrm{mM}$ glucose plus $40 \mathrm{mM}$ sodium acetate as carbon and energy sources. Yeast extract was supplemented in the culture of glucose plus acetate $\left(2 \mathrm{gl}^{-1}\right)$. Cells were collected in the exponential phase by centrifugation at $10,000 \mathrm{~g}$ at $4{ }^{\circ} \mathrm{C}$ for $20 \mathrm{~min}$. Cell pellets were washed twice in $100 \mathrm{mM}$ Tris- $\mathrm{HCl}$ ( $\mathrm{pH} 7.5), 1 \mathrm{mM}$ dithioerythreitol and suspended in $1 \mathrm{ml}$ of SDT-lysis buffer, which contained $100 \mathrm{mM}$ Tris- $\mathrm{HCl}(\mathrm{pH} 7.5), 4 \%$ SDS and $0.1 \mathrm{M}$ dithiotreitol. Protein extractions, separation, tryptic digestion and analysis were performed as described previously ${ }^{47}$. An Intestinimonas AF211 database downloaded from Uniprot (http://www.uniprot.org) was used together with a contaminant database that contains sequences of common contaminants; for instance, trypsin, keratin, bovine serum albumin. The proteomics result contained peptides and proteins with a false discovery rate of $<1 \%$ and proteins with at least two identified peptides of which should be unique and one should be unmodified without any reversed hits. The normal logarithm was taken from protein label-free quantitation (LFQ; normalized with respect to the total amount of protein and all of its identified peptides) intensities. Zero 'Log LFQ' values were replaced by a value of 5.4 (just below the lowest value) to make sensible ratio calculations possible. Relative protein quantitation of sample to control was done with Perseus 1.3.0.4 by applying a two sample $t$-test using the 'LFQ intensity' columns obtained with false discovery rate set to 0.05 and So set to 1 .

Preparation of Intestinimonas AF211 cell extract. Strain AF211 was grown in $150 \mathrm{ml}$ anaerobic bicarbonate-buffered medium containing $20 \mathrm{mM}$ lysine or $40 \mathrm{mM}$ glucose plus $40 \mathrm{mM}$ acetate as carbon and energy sources. Cells of strain A. rhamnosivorans DSM26241 (ref. 34) grown in $150 \mathrm{ml}$ anaerobic bicarbonatebuffered medium containing $20 \mathrm{mM}$ glucose were used as a negative control of the enzyme assay. Cells were collected in the exponential phase by centrifugation at $10,000 \mathrm{~g}$ at $4^{\circ} \mathrm{C}$ for $20 \mathrm{~min}$. Cell pellets were washed twice in an anaerobic buffer containing $100 \mathrm{mM}$ Tris- $\mathrm{HCl}$ (pH7.5), $1 \mathrm{mM}$ dithioerythreitol and suspended in $1 \mathrm{ml}$ of the same buffer. Cells were disrupted by sonication 5 times $\times 30 \mathrm{~s}$ and the cell suspension was cooled on ice for $30 \mathrm{~s}$ in between. Finally, the suspension was centrifuged for $10 \mathrm{~min}$ at $8,000 \mathrm{~g}$. The cell-free extract was transferred to serum bottles, flushed with $\mathrm{N}_{2}$ and either stored at $-20{ }^{\circ} \mathrm{C}$ or used directly for enzyme activity assays. All steps were performed in an anaerobic chamber with a $\mathrm{N}_{2} / \mathrm{H}_{2}$ (96:4; v/v) atmosphere, circulated over a palladium catalyst to eliminate traces of oxygen.

Detection of acetoacetyl-CoA transferase activity. CoA transferase activity was determined using a spectrophotometric assay ${ }^{19}$ at $310 \mathrm{~nm}, 25^{\circ} \mathrm{C}$ with $100 \mathrm{mM}$ Tris-Cl ( $\mathrm{pH} 8.1), 20 \mathrm{mM} \mathrm{MgCl}_{2}$ and $50 \mu \mathrm{M}$ butyryl-CoA plus $10 \mathrm{mM}$ lithium acetoacetate as substrates. For enzyme activity assay the formation of acetoacetyl$\operatorname{CoA}\left(E_{310 \mathrm{~nm}}=15.1 \mathrm{mM}^{-1} \mathrm{~cm}^{-1}\right)$ from butyryl-CoA and acetoacetate was followed by measuring the increase in $A_{310 \mathrm{~nm}}$ by means of an absorbance-recording spectrophotometer. Total volume of the mixture was $1.0 \mathrm{ml}$. The activities were expressed as $\mathrm{Umg}^{-1}$ of total proteins $\left(\mathrm{U}=\mu \mathrm{mol} \mathrm{min}{ }^{-1}\right)$. All assays were done with biological duplicates and 4-6 replicate measurements were performed. Total protein was quantified using Qubit2.0 Fluorometer (Invitrogen) according to the manufacturer's instructions.

Phylogenetic analysis of CoA transferase. For the construction of a CoA transferase phylogenetic tree, all butyryl-CoA:acetate CoA transferases (Ato) and butyryl-CoA:4-hydroxybutyrate CoA transferases (4Hbt) from known intestinal butyrate-producing bacteria according to refs. 28,48 were retrieved from the NCBI database (Fig. 4). Selected 4-hydroxybutyrate CoA transferases from Clostridium kluyveri, C. acetobutyricum and C. tetani were also included ${ }^{28}$. Butyryl-CoAacetoacetate CoA tranferases (Ato) from C. sticklandii DSM519, F. nucleatum 
ATCC25586 and C. acetobutylicum ATCC824 were collected from their genomes ${ }^{29,33,49}$. These CoA transferases are catalysing either butyrate or butanol formation. All amino-acid sequences of $4 \mathrm{Hbt}$ and AtoA/C/D from strain Intestinimonas AF211 were aligned with retrieved sequences using the CLUSTAL_X programme. A phylogenetic tree was constructed using the neighbour-joining algorithm by the MEGA 5 with 1,000 bootstraps to obtain confidence levels for the branches.

Quantification of Intestinimonas in the human colon. Stool samples of 10 Dutch healthy volunteers at different age and gender were collected (Supplementary Table 3). Genomic DNA was isolated via the bead-beating protocol described previously ${ }^{50}$. The $16 \mathrm{~S}$ rRNA sequences of phylogenetically related species were retrieved from GenBank (www.ncbi.nlm.nih.gov) and used to perform multiple alignments by using CLUSTALW. Primers for qPCR were designed using DNASTAR programme according to Walter et al. ${ }^{51}$ Primers were PFF590f: $5^{\prime}$-AAAACTATGGGCTCAACCCA-3' ${ }^{\prime}$ and PFF702r: $5^{\prime}$-GTCAGTTAAT GTCCAGCAGG- $3^{\prime}$ to quantify Intestinimonas AF211, which resulted in a 100-bp amplicon. Total bacteria were quantified using BAC1396F and PROK1492R primer pairs $^{52}$. The $16 \mathrm{~S}$ rRNA gene of strain AF211 was used for optimizing temperature and primer concentration, and for making standard curves. The programme that was used to amplify the partial 16S rRNA gene of strain AF211 was as following: $95^{\circ} \mathrm{C}$ for $5 \mathrm{~min}$ and 35 cycles consisting of $95^{\circ} \mathrm{C}$ for $30 \mathrm{~s}, 56.7^{\circ} \mathrm{C}$ for $10 \mathrm{~s}$ and $72^{\circ} \mathrm{C}$ for $30 \mathrm{~s} ; 95^{\circ} \mathrm{C}$ for $1 \mathrm{~min}$ and $60^{\circ} \mathrm{C}$ for $1 \mathrm{~min}$ and total bacteria as followed: $95^{\circ} \mathrm{C}$ for $10 \mathrm{~min}$ or $95^{\circ} \mathrm{C}$ for $20 \mathrm{~s}, 56.3^{\circ} \mathrm{C}$ for $30 \mathrm{~s}$ and $72^{\circ} \mathrm{C}$ for $30 \mathrm{~s} ; 95^{\circ} \mathrm{C}$ for $1 \mathrm{~min}$ and $60^{\circ} \mathrm{C}$ for $1 \mathrm{~min}$. Both were followed by a melting curve analysis. DNA copies were calculated from standard curves and subsequently used for the strain quantities.

Metagenome analysis. The metagenomic protein sequence data from faecal samples of 65 Human Microbiome Project subjects were obtained from MG-RAST (http://metagenomics.anl.gov/). The following samples were included: SRR063550, SRR063552, SRR063553, SRR063555, SRR063556, SRR063558, SRR063561, SRR063562, SRR063564, SRR063565, SRR061730, SRR063567, SRR063568, SRR063570, SRR063571, SRR063573, SRR063574, SRR063576, SRR063577, SRR063579, SRR063580, SRR063538, SRR063582, SRR063585, SRR063586, SRR063588, SRR063589, SRR063802, SRR063899, SRR063900, SRR063902, SRR063903, SRR063540, SRR063905, SRR063906, SRR063908, SRR063909, SRR063539, SRR063542, SRR063545, SRR063548, SRR063551, SRR063554 SRR063541, SRR063557, SRR063560, SRR063563, SRR063566, SRR063569, SRR063572, SRR063575, SRR063578, SRR063581, SRR063584, SRR063543, SRR063587, SRR063801, SRR063898, SRR063901, SRR063904, SRR063907, SRR063544, SRR063546, SRR063547 and SRR063549.

AF211 butyrogenic pathway protein homologues were searched from the metagenomic data by using Usearch v. 8.0.1517 with settings usearch_globa and id $=0.6$. The results were analysed in $\mathrm{R}$ v. 3.1.1 software environment (http://www.R-project.org) ${ }^{53}$.

\section{References}

1. Sommer, F. \& Bäckhed, F. The gut microbiota-masters of host development and physiology. Nat. Rev. Microbiol. 11, 227-238 (2013)

2. Qin, J. et al. A human gut microbial gene catalogue established by metagenomic sequencing. Nature 464, 59-65 (2010).

3. Hamer, H. M. et al. Review article: the role of butyrate on colonic function. Aliment. Pharmacol. Ther. 27, 104-119 (2008).

4. Rajilić-Stojanović, M. \& de Vos, W. M. The first 1000 cultured species of the human gastrointestinal microbiota. FEMS Microbiol. Rev. 38, 996-1047 (2014).

5. Flint, H. J., Scott, K. P., Ducan, S., Louis, P. \& Forano, E. Microbial degradation of complex carbohydrates in the gut. Gut Microbes 3, 289-306 (2012).

6. Qin, J. et al. A metagenome-wide association study of gut microbiota in type 2 diabetes. Nature 490, 55-60 (2012).

7. Scheppach, W., Luehrs, H. \& Menzel, T. Beneficial health effects of lowdigestible carbohydrate consumption. Br. J. Nutr. 85, S23-S30 (2001).

8. Duncan, S. H., Barcenilla, A., Stewart, C. S., Pryde, S. E. \& Flint, H. J. Acetate utilization and butyryl coenzyme A (CoA):acetate-CoA transferase in butyrateproducing bacteria from the human large intestine. Appl. Environ. Microbiol. 68, 5186-5190 (2002)

9. Belenguer, A. et al. Two routes of metabolic cross-feeding between Bifidobacterium adolescentis and butyrate-producing anaerobes from the human gut. Appl. Environ. Microbiol. 72, 3593-3599 (2006).

10. Barcenilla, A. et al. Phylogenetic relationships of butyrate-producing bacteria from the human gut. Appl. Environ. Microbiol. 66, 1654-1661 (2000).

11. Louis, P., Hold, G. L. \& Flint, H. J. The gut microbiota, bacterial metabolites and colorectal cancer. Nat. Rev. Microbiol. 12, 661-672 (2014).

12. Louis, P. et al. Restricted distribution of the butyrate kinase pathway among butyrate-producing bacteria from the human colon. J. Bacteriol. 186, 2099-2106 (2004).

13. Vital, M., Howe, A. C. \& Tiedje, J. M. Revealing the bacterial butyrate synthesis pathways by analyzing (meta)genomic data. MBio 5, e00889 (2014).
14. Deppe, V., Bongaerts, J., O’Connell, T., Maurer, K. H. \& Meinhardt, F. Enzymatic deglycation of Amadori products in bacteria: mechanisms, occurrence and physiological functions. Appl. Microbiol. Biotechnol. 90, 399-406 (2011).

15. Stadtman, T. C. \& White, F. H. Tracer studies on ornithine, lysine, and formate metabolism in an amino acid fermenting clostridium. J. Bacteriol. 67, 651-657 (1954).

16. Kreimeyer, A. et al. Identification of the last unknown genes in the fermentation pathway of lysine. J. Biol. Chem. 282, 7191-7197 (2007).

17. Yorifuji, T., Jeng, I. M. \& Barker, H. A. Purification and properties of 3-keto-5aminohexanoate cleavage enzyme from a lysine-fermenting clostridium. J. Biol. Chem. 252, 20-31 (1977).

18. Baker, J. J., Jeng, I. \& Barker, H. A. Purification and properties of l-erythro-3,5 diaminohexanoate dehydrogenase from a lysine-fermenting clostridium. J. Biol. Chem. 247, 7724-7734 (1972).

19. Barker, H. A. et al. Butyryl-CoA:acetoacetate CoA-transferase from a lysinefermenting clostridium. J. Biol. Chem. 253, 1219-1225 (1978).

20. Chirpich, T. P., Zappia, V., Costilow, R. N. \& Barker, H. A. Lysine 2,3aminomutase: purification and properties of a pyridoxal phosphate and S-adenosylmethionine-activated enzyme. J. Biol. Chem. 245, 1778-1789 (1970).

21. Kläring, K. et al. Intestinimonas butyriciproducens gen. nov., sp. nov., a butyrate-producing bacterium from the mouse intestine. Int. J. Syst. Evol. Microbiol. 63, 4606-4612 (2013).

22. Wiame, E. \& Van Schaftingen, E. Fructoselysine 3-epimerase, an enzyme involved in the metabolism of the unusual Amadori compound psicoselysine in Escherichia coli. Biochem. J. 378, 1047-1052 (2004).

23. Wiame, E., Delpierre, G., Collard, F. \& Van Schaftingen, E. Identification of a pathway for the utilization of the Amadori product fructoselysine in Escherichia coli. J. Biol. Chem. 277, 42523-42529 (2002).

24. Plugge, C. M., van Leeuwen, J., Hummelen, T., Balk, M. \& Stams, A. J. M. Elucidation of the pathways of catabolic glutamate conversion in three thermophilic anaerobic bacteria. Arch. Microbiol. 176, 29-36 (2001).

25. Herrmann, G., Jayamani, E., Mai, G. \& Buckel, W. Energy conservation via electron-transferring flavoprotein in anaerobic bacteria. J. Bacteriol. 190, 784-791 (2008).

26. Li, F. et al. Coupled ferredoxin and crotonyl coenzyme A (CoA) reduction with $\mathrm{NADH}$ catalyzed by the butyryl-CoA dehydrogenase/Etf complex from Clostridium kluyveri. J. Bacteriol. 190, 843-850 (2008).

27. Boynton, Z. L., Bennet, G. N. \& Rudolph, F. B. Cloning, sequencing, and expression of clustered genes encoding beta-hydroxybutyryl-coenzyme A (CoA) dehydrogenase, crotonase, and butyryl-CoA dehydrogenase from Clostridium acetobutylicum ATCC 824. J. Bacteriol. 178, 3015-3024 (1996).

28. Charrier, C. et al. A novel class of CoA-transferase involved in short-chain fatty acid metabolism in butyrate-producing human colonic bacteria. Microbiology 152, 179-185 (2006).

29. Fonknechten, N. et al. Clostridium sticklandii, a specialist in amino acid degradation:revisiting its metabolism through its genome sequence. $B M C$ Genomics 11, 1-12 (2010).

30. Wiesenborn, D. P., Rudolph, F. B. \& Papoutsakis, E. T. Coenzyme A transferase from Clostridium acetobutylicum ATCC 824 and its role in the uptake of acids. Appl. Environ. Microbiol. 55, 323-329 (1989).

31. Bennett, G. N. \& Rudolph, F. B. The central metabolic pathway from acetylCoA to butyryl-CoA in Clostridium acetobutylicum. FEMS Microbiol. Rev. 17, 241-249 (1995)

32. Barker, H. A., Kahn, J. M. \& Hedrick, L. Pathway of lysine degradation in Fusobacterium nucleatum. J. Bacteriol. 152, 201-207 (1982).

33. Kapatral, V. et al. Genome sequence and analysis of the oral bacterium Fusobacterium nucleatum strain ATCC 25586. J. Bacteriol. 184, 2005-2018 (2002).

34. Bui, T. P. N., de Vos, W. M. \& Plugge, C. M. Anaerostipes rhamnosivorans sp. nov., a human intestinal, butyrate-forming bacterium. Int. J. Syst. Evol. Microbiol. 64, 787-793 (2014).

35. The Human Microbiome Project Consortium. Structure, function and diversity of the healthy human microbiome. Nature 486, 207-214 (2012).

36. Rettedal, E. A., Gumpert, H. \& Sommer, M. O. A. Cultivation-based multiplex phenotyping of human gut microbiota allows targeted recovery of previously uncultured bacteria. Nat. Commun. 5, 4714 (2014).

37. Leiros, H. K. et al. Trypsin specificity as elucidated by LIE calculations, X-ray structures, and association constant measurements. Protein Sci. 13, 1056-1070 (2004).

38. Rogers, A. H., Chen, J., Zilm, P. S. \& Gully, N. J. The behaviour of Fusobacterium nucleatum chemostat-grown in glucose- and amino acid-based chemically defined media. Anaerobe 4, 111-116 (1998).

39. Windey, K. et al. Modulation of protein fermentation does not affect fecal water toxicity: a randomized cross-over study in healthy subjects. PLoS ONE 7, e52387 (2012).

40. Brownlee, M. Glycation and diabetic complications. Diabetes 43, 836-841 (1994). 
41. Baynes, J. W. \& Thorpe, S. R. Glycoxidation and lipoxidation in atherogenesis. Free Radic. Biol. Med. 28, 1708-1716 (2000).

42. West, R. K. et al. Dietary advanced glycation end products are associated with decline in memory in young elderly. Mech. Ageing Dev. 140, 10-12 (2014)

43. Cai, W. et al. Oral glycotoxins are a modifiable cause of dementia and the metabolic syndrome in mice and humans. Proc. Natl Acad. Sci. USA 111, 4940-4945 (2014).

44. Stams, A. J. M., Van Dijk, J. B., Dijkema, C. \& Plugge, C. M. Growth of syntrophic propionate-oxidizing bacteria with fumarate in the absence of methanogenic bacteria. Appl. Environ. Microbiol. 59, 1114-1119 (1993).

45. Zwietering, M. H., Jongenburger, I., Rombouts, F. M. \& Van 't Riet, K. Modeling of the bacterial growth curve. Appl. Environ. Microbiol. 56, 1875-1881 (1990).

46. van Gelder, A. H., Aydin, R., Alves, M. M. \& Stams, A. J. M. 1,3-Propanediol production from glycerol by a newly isolated Trichococcus strain. Microbiol. Biotechnol. 5, 573-578 (2012).

47. Oosterkamp, M. J., Boeren, S., Plugge, C. M., Schaap, P. J. \& Stams, A. J. M. Metabolic response of Alicycliphilus denitrificans strain BC toward electron acceptor variation. Proteomics 13, 2886-2894 (2013).

48. Louis, P. \& Flint, H. J. Diversity, metabolism and microbial ecology of butyrateproducing bacteria from the human large intestine. FEMS Microbiol. Lett. 294, 1-8 (2009).

49. Gerischer, U. \& Dürre, P. Cloning, sequencing, and molecular analysis of the acetoacetate decarboxylase gene region from Clostridium acetobutylicum. J. Bacteriol. 172, 6907-6918 (1990).

50. Salonen, A. et al. Comparative analysis of fecal DNA extraction methods with phylogenetic microarray: Effective recovery of bacterial and archaeal DNA using mechanical cell lysis. J. Microbiol. Methods 81, 127-134 (2010).

51. Walter, J. et al. Detection of Lactobacillus, Pediococcus, Leuconostoc, and Weissella species in human feces by using group-specific PCR primers and denaturing gradient gel electrophoresis. Appl. Environ. Microbiol. 67, 2578-2585 (2001)

52. Suzuki, M. T., Taylor, L. T. \& DeLong, E. F. Quantitative analysis of smallsubunit rRNA genes in mixed microbial populations via $5^{\prime}$-nuclease assays. Appl. Environ. Microbiol. 66, 4605-4614 (2000).

53. Edgar, R. C. Search and clustering orders of magnitude faster than BLAST. Bioinformatics 26, 2460-2461 (2010).

\section{Acknowledgements}

This research has partly been supported by grant 250172-Microbes Inside of the European Research Council to W.M.d.V. and the Soehngen Institute of Anaerobic Microbiology, funded by the Netherlands Organization for Scientific Research. We thank Dr Erwin Zoetendal and Prof. Stephen O'Keefe for providing faecal samples used in this study, Dr Elsa Wiame for the kind gift of fructoselysine, Prof. Fons Stams for helpful discussions, Ton van Gelder for technical support and Mark Davids for sharing computational infrastructure.

\section{Author contributions}

W.M.d.V., C.M.P. and T.P.N.B. designed the research; T.P.N.B. performed the research; J.R. contributed to the (meta)genome analyses; S.B. performed the liquid chromatography-mass spectrometry/mass spectrometry and P.d.W. contributed to the ${ }^{13}$ C-NMR and HBMC analysis; T.P.N.B., J.R., S.B., P.d.W., C.M.P. and W.M.d.V. analysed the data; and T.P.N.B., C.M.P. and W.M.d.V. wrote the paper.

\section{Additional information}

Supplementary Information accompanies this paper at http://www.nature.com/ naturecommunications

Competing financial interests: The authors declare no competing financial interests.

Reprints and permission information is available online at http://npg.nature.com/ reprintsandpermissions/

How to cite this article: Bui, T.P.N. et al. Production of butyrate from lysine and the Amadori product fructoselysine by a human gut commensal. Nat. Commun. 6:10062 doi: $10.1038 /$ ncomms10062 (2015).

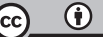

This work is licensed under a Creative Commons Attribution 4.0 International License. The images or other third party material in this article are included in the article's Creative Commons license, unless indicated otherwise in the credit line; if the material is not included under the Creative Commons license, users will need to obtain permission from the license holder to reproduce the material. To view a copy of this license, visit http://creativecommons.org/licenses/by/4.0/ 